\title{
SOLUTION OF THE DISPERSIONLESS HIROTA EQUATIONS
}

\author{
R. Carroll* and Y. Kodama ${ }^{\dagger}$ \\ May, 1995
}

\begin{abstract}
The dispersionless differential Fay identity is shown to be equivalent to a kernel expansion providing a universal algebraic characterization and solution of the dispersionless Hirota equations. Some calculations based on D-bar data of the action are also indicated.
\end{abstract}

\section{Introduction}

The dKP hierarchy (= dispersionless limit of the KP hierarchy) and various reductions thereof (such as dNKdV) play an increasingly important role in topological field theory and its connections to strings and 2-D gravity (cf. [2, 3, $1,6,6,10,12,21,24,25)$ ). There are also connections to twistor theory in the spirit of [24, 25] but we do not pursue this direction here. We address rather the dispersionless Hirota equations which arise from the dispersionless differential Fay identity and characterize dKP. We show that this identity is equivalent to a kernel expansion which generates the dispersionless Hirota equations in a universal algebraic manner depending only on the expressions $\lambda=P+\sum_{1}^{\infty} U_{n+1} P^{-n}$ or equivalently $P=\lambda-\sum_{1}^{\infty} P_{j+1} \lambda^{-j}$. The $P_{j+1}$ play the role of universal coordinates and this aspect has important consequences in topological field theory (cf. 䧃). The solutions are given in terms of a residue formula $F_{m n}=\operatorname{Res}_{P}\left[\lambda^{m} d \lambda_{+}^{n}\right]$, which in fact are shown to be generated by the universal coordinates $P_{n+1}=F_{1 n} / n$. We also show how the Hamilton-Jacobi and hodograph analysis for dNKdV yields D-bar data for the action $S$ and this gives a moment type

${ }^{*}$ Department of mathematics, University of Illinois, Urbana, IL 61801

E-mail: rcarroll@math.uiuc.edu

${ }^{\dagger}$ Department of Mathematics, Ohio State University, Columbus, OH 43210

E-mail: kodama@math.ohio-state.edu 
formula for the $F_{j}$ in terms of $\bar{\partial} S$. In the dKdV situation this actually yields a direct formula for the $F_{i j}$ providing an immediate alternative method of calculation.

\section{Background on KP and dKP}

One can begin with two pseudodifferential operators $(\partial=\partial / \partial x)$,

$$
L=\partial+\sum_{1}^{\infty} u_{n+1} \partial^{-n} ; \quad W=1+\sum_{1}^{\infty} w_{n} \partial^{-n}
$$

called the Lax operator and gauge operator respectively, where the generalized Leibnitz rule with $\partial^{-1} \partial=\partial \partial^{-1}=1$ applies

$$
\partial^{i} f \cdot=\sum_{j=0}^{\infty}\left(\begin{array}{l}
i \\
j
\end{array}\right)\left(\partial^{j} f\right) \partial^{i-j}
$$

for any $i \in \mathbf{Z}$, and $L=W \partial W^{-1}$. The KP hierarchy then is determined by the Lax equations $\left(\partial_{n}=\partial / \partial t_{n}\right)$,

$$
\partial_{n} L=\left[B_{n}, L\right]:=B_{n} L-L B_{n},
$$

where $B_{n}=L_{+}^{n}$ is the differential part of $L^{n}=L_{+}^{n}+L_{-}^{n}=\sum_{0}^{\infty} \ell_{i}^{n} \partial^{i}+\sum_{-\infty}^{-1} \ell_{i}^{n} \partial^{i}$. One can also express this via the Sato equation,

$$
\partial_{n} W W^{-1}=-L_{-}^{n},
$$

which is particularly well adapted to the dKP theory. Now define the wave function via

$$
\psi=W e^{\xi}=w(t, \lambda) e^{\xi} ; \quad \xi:=\sum_{1}^{\infty} t_{n} \lambda^{n} ; \quad w(t, \lambda)=1+\sum_{1}^{\infty} w_{n}(t) \lambda^{-n},
$$

where $t_{1}=x$. There is also an adjoint wave function $\psi^{*}=W^{*-1} \exp (-\xi)=$ $w^{*}(t, \lambda) \exp (-\xi), w^{*}(t, \lambda)=1+\sum_{1}^{\infty} w_{i}^{*}(t) \lambda^{-i}$, and one has equations

$$
L \psi=\lambda \psi ; \quad \partial_{n} \psi=B_{n} \psi ; \quad L^{*} \psi^{*}=\lambda \psi^{*} ; \quad \partial_{n} \psi^{*}=-B_{n}^{*} \psi^{*} .
$$

Note that the KP hierarchy (2.3) is then given by the compatibility conditions among these equations with an iso-spectral property. Next one has the fundamental tau function $\tau(t)$ and vertex operators $\mathbf{X}, \mathbf{X}^{*}$ satisfying

$$
\psi(t, \lambda)=\frac{\mathbf{X}(\lambda) \tau(t)}{\tau(t)}=\frac{e^{\xi} G_{-}(\lambda) \tau(t)}{\tau(t)}=\frac{e^{\xi} \tau\left(t-\left[\lambda^{-1}\right]\right)}{\tau(t)} ;
$$




$$
\psi^{*}(t, \lambda)=\frac{\mathbf{X}^{*}(\lambda) \tau(t)}{\tau(t)}=\frac{e^{-\xi} G_{+}(\lambda) \tau(t)}{\tau(t)}=\frac{e^{-\xi} \tau\left(t+\left[\lambda^{-1}\right]\right)}{\tau(t)},
$$

where $G_{ \pm}(\lambda)=\exp \left( \pm \xi\left(\tilde{\partial}, \lambda^{-1}\right)\right)$ with $\tilde{\partial}=\left(\partial_{1},(1 / 2) \partial_{2},(1 / 3) \partial_{3}, \cdots\right)$ and $t \pm\left[\lambda^{-1}\right]=$ $\left(t_{1} \pm \lambda^{-1}, t_{2} \pm(1 / 2) \lambda^{-2}, \cdots\right)$. One writes also

$$
e^{\xi}:=\exp \left(\sum_{1}^{\infty} t_{n} \lambda^{n}\right)=\sum_{0}^{\infty} \chi_{j}\left(t_{1}, t_{2}, \cdots, t_{j}\right) \lambda^{j}
$$

where the $\chi_{j}$ are the elementary Schur polynomials, which arise in many important formulas (cf. below).

We mention now the famous bilinear identity which generates the entire KP hierarchy. This has the form

$$
\oint_{\infty} \psi(t, \lambda) \psi^{*}\left(t^{\prime}, \lambda\right) d \lambda=0
$$

where $\oint_{\infty}(\cdot) d \lambda$ is the residue integral about $\infty$, which we also denote $\operatorname{Res}_{\lambda}[(\cdot) d \lambda]$. Using (2.7) this can also be written in terms of tau functions as

$$
\oint_{\infty} \tau\left(t-\left[\lambda^{-1}\right]\right) \tau\left(t^{\prime}+\left[\lambda^{-1}\right]\right) e^{\xi(t, \lambda)-\xi\left(t^{\prime}, \lambda\right)} d \lambda=0
$$

This leads to the characterization of the tau function in bilinear form expressed via $\left(t \rightarrow t-y, t^{\prime} \rightarrow t+y\right)$

$$
\left(\sum_{0}^{\infty} \chi_{n}(-2 y) \chi_{n+1}(\tilde{D}) e^{\sum_{1}^{\infty} y_{i} D_{i}}\right) \tau \cdot \tau=0
$$

where $D_{i}$ is the Hirota derivative defined as $D_{j}^{m} a \cdot b=\left.\left(\partial^{m} / \partial s_{j}^{m}\right) a\left(t_{j}+s_{j}\right) b\left(t_{j}-s_{j}\right)\right|_{s=0}$ and $\tilde{D}=\left(D_{1},(1 / 2) D_{2},(1 / 3) D_{3}, \cdots\right)$. In particular, we have from the coefficients of $y_{n}$ in (2.11),

$$
D_{1} D_{n} \tau \cdot \tau=2 \chi_{n+1}(\tilde{D}) \tau \cdot \tau
$$

which are called the Hirota bilinear equations. Such calculations with vertex operator equations and residues, in the context of finite zone situations where the tau function is intimately related to theta functions, also led historically to the Fay trisecant identity, which can be expressed generally as the Fay identity via (cf. [2, 8] - c.p. means cyclic permutations)

$$
\sum_{c . p .}\left(s_{0}-s_{1}\right)\left(s_{2}-s_{3}\right) \tau\left(t+\left[s_{0}\right]+\left[s_{1}\right]\right) \tau\left(t+\left[s_{2}\right]+\left[s_{3}\right]\right)=0 .
$$

This can be also derived from the bilinear identity (2.10). Differentiat ing this in $s_{0}$, then setting $s_{0}=s_{3}=0$, then dividing by $s_{1} s_{2}$, and finally shifting $t \rightarrow t-\left[s_{2}\right]$, leads 
to the differential Fay identity,

$$
\begin{aligned}
& \tau(t) \partial \tau\left(t+\left[s_{1}\right]-\left[s_{2}\right]\right)-\tau\left(t+\left[s_{1}\right]-\left[s_{2}\right]\right) \partial \tau(t) \\
& =\left(s_{1}^{-1}-s_{2}^{-1}\right)\left[\tau\left(t+\left[s_{1}\right]-\left[s_{2}\right]\right) \tau(t)-\tau\left(t+\left[s_{1}\right]\right) \tau\left(t-\left[s_{2}\right]\right)\right] .
\end{aligned}
$$

The Hirota equations (2.12) can be also derived from (2.14) by taking the limit $s_{1} \rightarrow s_{2}$. The identity (2.14) will play an important role later.

Now for the dispersionless theory (dKP) one can think of fast and slow variables, etc., or averaging procedures, but simply one takes $t_{n} \rightarrow \epsilon t_{n}=T_{n}\left(t_{1}=x \rightarrow \epsilon x=X\right)$ in the KP equation $u_{t}=(1 / 4) u_{x x x}+3 u u_{x}+(3 / 4) \partial^{-1} u_{y y},\left(y=t_{2}, t=t_{3}\right)$, with $\partial_{n} \rightarrow \epsilon \partial / \partial T_{n}$ and $u\left(t_{n}\right) \rightarrow U\left(T_{n}\right)$ to obtain $\partial_{T} U=3 U U_{X}+(3 / 4) \partial^{-1} U_{Y Y}$ when $\epsilon \rightarrow 0(\partial=\partial / \partial X$ now $)$. Thus the dispersion term $u_{x x x}$ is removed. In terms of hierarchies we write

$$
L_{\epsilon}=\epsilon \partial+\sum_{1}^{\infty} u_{n+1}(T / \epsilon)(\epsilon \partial)^{-n}
$$

and think of $u_{n}(T / \epsilon)=U_{n}(T)+O(\epsilon)$, etc. One takes then a WKB form for the wave function with the action $S$ [17],

$$
\psi=\exp \left[\frac{1}{\epsilon} S(T, \lambda)\right]
$$

Replacing now $\partial_{n}$ by $\epsilon \partial_{n}$, where $\partial_{n}=\partial / \partial T_{n}$ now, we define $P:=\partial S=S_{X}$. Then $\epsilon^{i} \partial^{i} \psi \rightarrow P^{i} \psi$ as $\epsilon \rightarrow 0$ and the equation $L \psi=\lambda \psi$ becomes

$$
\lambda=P+\sum_{1}^{\infty} U_{n+1} P^{-n} ; \quad P=\lambda-\sum_{1}^{\infty} P_{i+1} \lambda^{-i}
$$

where the second equation is simply the inversion of the first. We also note from $\partial_{n} \psi=B_{n} \psi=\sum_{0}^{n} b_{n m}(\epsilon \partial)^{m} \psi$ that one obtains $\partial_{n} S=\mathcal{B}_{n}(P)=\lambda_{+}^{n}$ where the subscript $(+)$ refers now to powers of $P$ (note $\epsilon \partial_{n} \psi / \psi \rightarrow \partial_{n} S$ ). Thus $B_{n}=L_{+}^{n} \rightarrow \mathcal{B}_{n}(P)=$ $\lambda_{+}^{n}=\sum_{0}^{n} b_{n m} P^{m}$ and the KP hierarchy goes to

$$
\partial_{n} P=\partial \mathcal{B}_{n}
$$

which is the dKP hierarchy (note $\partial_{n} S=\mathcal{B}_{n} \Rightarrow \partial_{n} P=\partial \mathcal{B}_{n}$ ). The action $S$ in $(2.16)$ can be computed from (2.7) in the limit $\epsilon \rightarrow 0$ as

$$
S=\sum_{1}^{\infty} T_{n} \lambda^{n}-\sum_{1}^{\infty} \frac{\partial_{m} F}{m} \lambda^{-m}
$$

where the function $F=F(T)$ (free energy) is defined by 24

$$
\tau=\exp \left[\frac{1}{\epsilon^{2}} F(T)\right]
$$


The formula (2.19) then solves the dKP hierarchy (2.18), i.e. $P=\mathcal{B}_{1}=\partial S$ and

$$
\mathcal{B}_{n}=\partial_{n} S=\lambda^{n}-\sum_{1}^{\infty} \frac{F_{n m}}{m} \lambda^{-m},
$$

where $F_{n m}=\partial_{n} \partial_{m} F$ which play an important role in the theory of dKP.

Now following [25] we write the differential Fay identity (2.14) with $\epsilon \partial_{n}$ replacing $\partial_{n}$ etc. in the form

$$
\begin{aligned}
& \frac{\tau\left(T-\epsilon\left[\mu^{-1}\right]-\epsilon\left[\lambda^{-1}\right]\right) \tau(T)}{\tau\left(T-\epsilon\left[\mu^{-1}\right]\right) \tau\left(T-\epsilon\left[\lambda^{-1}\right]\right)} \\
& =1+\frac{\epsilon \partial}{\mu-\lambda}\left[\log \left(\tau\left(T-\epsilon\left[\mu^{-1}\right]\right)\right)-\log \left(\tau\left(T-\epsilon\left[\lambda^{-1}\right]\right)\right)\right]
\end{aligned}
$$

(in (2.14) take $t \rightarrow t-\left[s_{1}\right], s_{1}=\mu^{-1}, s_{2}=\lambda^{-1}$ and insert $\epsilon$ at the appropriate places; note $T$ is used in (2.22)) . One notes from (2.8) that $\exp \left(-\xi\left(\tilde{\partial}, \lambda^{-1}\right)\right)=\sum_{1}^{\infty} \chi_{j}(\tilde{\partial}) \lambda^{-j}$ so taking logarithms in (2.22) and using (2.20) yield

$$
\begin{aligned}
& \frac{1}{\epsilon^{2}} \sum_{m, n=1}^{\infty} \mu^{-m} \lambda^{-n} \chi_{n}(-\epsilon \tilde{\partial}) \chi_{m}(-\epsilon \tilde{\partial}) F \\
& =\log \left[1+\frac{1}{\epsilon} \sum_{1}^{\infty} \frac{\mu^{-n}-\lambda^{-n}}{\mu-\lambda} \chi_{n}(-\epsilon \tilde{\partial}) \partial_{X} F\right] .
\end{aligned}
$$

In passing this to limits only the second order derivatives survive, and one gets the dispersionless differential Fay identity (note $\chi_{n}(-\epsilon \tilde{\partial})$ contributes here only $-\epsilon \partial_{n} / n$ )

$$
\sum_{m, n=1}^{\infty} \mu^{-m} \lambda^{-n} \frac{F_{m n}}{m n}=\log \left(1-\sum_{1}^{\infty} \frac{\mu^{-n}-\lambda^{-n}}{\mu-\lambda} \frac{F_{1 n}}{n}\right) .
$$

Although (2.24) only uses a subset of the Plücker relations defining the KP hierarchy it was shown in [25] that this subset is sufficient to determine KP; hence (2.24) characterizes the function $F$ for dKP. Following [7], we now derive a dispersionless limit of the Hirota bilinear equations (2.12), which we call the dispersionless Hirota equations. We first note from (2.19) and (2.17) that $F_{1 n}=n P_{n+1}$ so

$$
\sum_{1}^{\infty} \lambda^{-n} \frac{F_{1 n}}{n}=\sum_{1}^{\infty} P_{n+1} \lambda^{-n}=\lambda-P(\lambda) .
$$

Consequently the right side of $(2.24)$ becomes $\log \left[\frac{P(\mu)-P(\lambda)}{\mu-\lambda}\right]$ and for $\mu \rightarrow \lambda$ with $\dot{P}:=\partial_{\lambda} P$ we have

$$
\log \dot{P}(\lambda)=\sum_{m, n=1}^{\infty} \lambda^{-m-n} \frac{F_{m n}}{m n}=\sum_{j=1}^{\infty}\left(\sum_{n+m=j} \frac{F_{m n}}{m n}\right) \lambda^{-j} .
$$


Then using the elementary Schur polynomial defined in (2.8) and (2.17), we obtain

$$
\begin{aligned}
\dot{P}(\lambda) & =\sum_{0}^{\infty} \chi_{j}\left(Z_{2}, \cdots, Z_{j}\right) \lambda^{-j} \\
& =1+\sum_{1}^{\infty} j P_{j+1} \lambda^{-j-1}=1+\sum_{1}^{\infty} F_{1 j} \lambda^{-j-1},
\end{aligned}
$$

where $Z_{i}, i \geq 2$ are defined by

$$
Z_{i}=\sum_{m+n=i} \frac{F_{m n}}{m n} .
$$

Note that $Z_{1}=0$ is assumed for the polynomials $\chi_{j}$. Thus we obtain the dispersionless Hirota equations,

$$
F_{1 j}=\chi_{j+1}\left(Z_{2}, \cdots, Z_{j+1}\right) .
$$

These can be also derived directly from (2.12) with (2.20) in the limit $\epsilon \rightarrow 0$ or by expanding (2.26) in powers of $\lambda^{-n}$. We list here a few entries from such an expansion (cf. [7]):

$$
\begin{aligned}
\lambda^{-4}: & \frac{1}{2} F_{11}^{2}-\frac{1}{3} F_{13}+\frac{1}{4} F_{22}=0 \\
\lambda^{-5}: & F_{11} F_{12}-\frac{1}{2} F_{14}+\frac{1}{3} F_{23}=0 \\
\lambda^{-6}: & \frac{1}{3} F_{11}^{3}-\frac{1}{2} F_{12}^{2}-F_{11} F_{13}+\frac{3}{5} F_{15}-\frac{1}{9} F_{33}-\frac{1}{4} F_{24}=0 \\
\lambda^{-7}: & F_{11}^{2} F_{12}-F_{12} F_{13}-F_{11} F_{14}+\frac{2}{3} F_{16}-\frac{1}{6} F_{34}-\frac{1}{5} F_{25}=0 \\
\lambda^{-8}: & \frac{1}{4} F_{11}^{4}-F_{11} F_{12}^{2}-F_{11}^{2} F_{13}+\frac{1}{2} F_{13}^{2}+F_{12} F_{14}+ \\
& +F_{11} F_{15}-\frac{5}{7} F_{17}+\frac{1}{16} F_{44}+\frac{2}{15} F_{35}+\frac{1}{6} F_{26}=0 \\
\lambda^{-9}: & F_{11}^{3} F_{12}-\frac{1}{3} F_{12}^{3}-2 F_{11} F_{12} F_{13}-F_{11}^{2} F_{14}+F_{13} F_{14} \\
& +F_{12} F_{15}+F_{11} F_{16}-\frac{3}{4} F_{18}+\frac{1}{10} F_{45}+\frac{1}{9} F_{36}+\frac{1}{7} F_{27}=0 \\
\lambda^{-10}: & \frac{1}{5} F_{11}^{5}-\frac{3}{2} F_{11}^{2} F_{12}^{2}-F_{11}^{3} F_{13}+F_{12}^{2} F_{13}+F_{11} F_{13}^{2} \\
& +2 F_{11} F_{12} F_{14}-\frac{1}{2} F_{14}^{2}+F_{11}^{2} F_{15}-F_{13} F_{15}-F_{12} F_{16}-F_{11} F_{17} \\
& +\frac{7}{9} F_{19}-\frac{1}{25} F_{55}-\frac{1}{12} F_{46}-\frac{2}{21} F_{37}-\frac{1}{8} F_{28}=0 .
\end{aligned}
$$


These equations are discussed in various ways below and we will also show the equivalence of the dispersionless Fay differential identity with another formula of a Cauchy kernel in Section 3. Note here that for $U=F_{11}$ the first equation in (2.30) is a dKP equation $U_{T}=3 U U_{X}+(3 / 4) \partial^{-1} U_{Y Y}$ and other equations in the hierarchy are similarly generated.

It is also interesting to note that the dispersionless Hirota equations (2.29) or (2.30) can be regarded as algebraic equations for "symbols" $F_{m n}$, which are defined via (2.21), i.e.

$$
\mathcal{B}_{n}:=\lambda_{+}^{n}=\lambda^{n}-\sum_{1}^{\infty} \frac{F_{n m}}{m} \lambda^{-m} .
$$

Lemma 1 The symbols satisfy

$$
F_{n m}=F_{m n}=\operatorname{Res}_{P}\left[\lambda^{m} d \lambda_{+}^{n}\right] .
$$

Proof. One need simply observe that

$$
\begin{aligned}
F_{n m} & =-\operatorname{Res}_{\lambda}\left[\mathcal{B}_{n} d \lambda^{m}\right]=-\operatorname{Res}_{P}\left[\mathcal{B}_{n} d \lambda^{m}\right] \\
& =-\operatorname{Res}_{P}\left[\lambda_{+}^{n} d \lambda_{-}^{m}\right]=-\operatorname{Res}_{P}\left[\lambda^{n} d \lambda_{-}^{m}\right] \\
& =\operatorname{Res}_{P}\left[\lambda^{n} d \lambda_{+}^{m}\right]=\operatorname{Res}_{P}\left[\lambda^{m} d \lambda_{+}^{n}\right]=F_{m n} .
\end{aligned}
$$

Here we have used $\lambda_{-}^{m}=\lambda^{m}-\lambda_{+}^{m}$ and $\operatorname{Res}[d(a b)]=0=\operatorname{Res}[d a b+a d b]$ for pseudodifferential or formal Laurent expansions $a$ and $b$.

Thus we have:

Theorem 1 For $\lambda, P$ given algebraically as in (2.17), with no a priori connection to $d K P$, and for $\mathcal{B}_{n}$ defined as in the last equation of (2.31) via a formal collection of the symbols with two indices $F_{m n}$, it follows that the dispersionless Hirota equations (2.29) or 2.39) are nothing but the polynomial identities among $F_{m n}$.

In the next section we give a direct proof of this fact, that is, the $F_{m n}$ defined in (2.31) satisfy the dispersionless Hirota equations, which we designate by (2.29) in what follows.

Now one very natural way of developing dKP begins with (2.17) and (2.18) since eventually the $P_{j+1}$ can serve as universal coordinates (cf. here [4] for a discussion of this in connection with topological field theory $=$ TFT). This point of view is also natural in terms of developing a Hamilton-Jacobi theory involving ideas from the hodograph - Riemann invariant approach (cf. [6, 15, 17, 18] and (4.18) below) and in connecting NKdV ideas to TFT, strings, and quantum gravity (cf. [10] for a 
survey of this). It is natural here to work with $Q_{n}:=(1 / n) \mathcal{B}_{n}$ and note that $\partial_{n} S=\mathcal{B}_{n}$ corresponds to $\partial_{n} P=\partial \mathcal{B}_{n}=n \partial Q_{n}$. In this connection one often uses different time variables, say $T_{n}^{\prime}=n T_{n}$, so that $\partial_{n}^{\prime} P=\partial Q_{n}$, and $G_{m n}=F_{m n} / m n$ is used in place of $F_{m n}$. Here however we will retain the $T_{n}$ notation with $\partial_{n} S=n Q_{n}$ and $\partial_{n} P=n \partial Q_{n}$ since one will be connecting a number of formulas to standard KP notation. Now given (2.17) and (2.18) the equation $\partial_{n} P=n \partial Q_{n}$ corresponds to Benney's moment equations and is equivalent to a system of Hamiltonian equations defining the dKP hierarchy (cf. [6, 18] and remarks after (2.18); the Hamilton-Jacobi equations are $\partial_{n} S=n Q_{n}$ with Hamiltonians $\left.n Q_{n}(X, P=\partial S)\right)$.

We now have an important formula for the functions $Q_{n}$ :

Proposition 1 [18] The generating function of $\partial_{P} Q_{n}(\lambda)$ is given by

$$
\frac{1}{P(\mu)-P(\lambda)}=\sum_{1}^{\infty} \partial_{P} Q_{n}(\lambda) \mu^{-n} .
$$

Proof. Multiplying (2.17) by $\lambda^{n-1} \partial_{P} \lambda$, we have

$$
\lambda^{n} \partial_{P} \lambda=P(\lambda) \lambda^{n-1} \partial_{P} \lambda+P_{2} \lambda^{n-2} \partial_{P} \lambda+\cdots .
$$

Taking the polynomial part leads to a recurrence relation

$$
\partial_{P} Q_{n+1}(\lambda)=P(\lambda) \partial_{P} Q_{n}(\lambda)+P_{2} \partial_{P} Q_{n-1}(\lambda)+\cdots+P_{n} \partial_{P} Q_{1}(\lambda) .
$$

Then noting $\partial_{P} Q_{1}=1, Q_{0}=1$, and summing up (2.36) as follows, we obtain

$$
\begin{aligned}
& \sum_{n=0}^{\infty}\left(\partial_{P} Q_{n+1}(\lambda)-P(\lambda) \partial_{P} Q_{n}(\lambda)-\sum_{i+j=n} P_{j+1} \partial_{P} Q_{i}(\lambda)\right) \mu^{-n} \\
& =\left(\mu-P(\lambda)-\sum_{1}^{\infty} P_{j+1} \mu^{-j}\right) \sum_{1}^{\infty} \partial_{P} Q_{i}(\lambda) \mu^{-i}=1,
\end{aligned}
$$

which is just (2.34).

This is a very important kernel formula which will come up in various ways in what follows. In particular we note

$$
\oint_{\infty} \frac{\mu^{n}}{P(\mu)-P(\lambda)} d \mu=\partial_{P} Q_{n+1}(\lambda)
$$

which gives a key formula in the Hamilton-Jacobi method for the dKP 18. Thus it represents a Cauchy kernel and has a version on Riemann surfaces related to the prime form (cf. [19]). In fact the kernel is a dispersionless limit of the Fay prime form. Also note here that the function $P(\lambda)$ alone provides all the information necessary for the dKP theory. This will be discussed further in the next section. 


\section{Solutions and variations}

We have already discussed solutions of the dispersionless Hirota equations (2.29) briefly in Theorem 1, but we go now to some different points of view. First let us prove:

Theorem 2 The kernel formula (2.34) is equivalent to the dispersionless differential Fay identity (2.24).

This will follow from the following lemma.

Lemma 2 Let $\chi_{n}\left(Q_{1}, \cdots, Q_{n}\right)$ denote the elementary Schur polynomials defined as in (2.8). Then

$$
\partial_{P} Q_{n}=\chi_{n-1}\left(Q_{1}, \cdots, Q_{n-1}\right) .
$$

Proof. We integrate the kernel formula (2.34) with respect to $P(k)$, and normalize at $\lambda=\infty$ to obtain

$$
\frac{1}{P(\lambda)-P(k)}=\frac{1}{\lambda} \exp \left(\sum_{1}^{\infty} Q_{n}(k) \lambda^{-n}\right)=\sum_{1}^{\infty} \chi_{m-1}\left(Q_{1}, \cdots, Q_{m-1}\right) \lambda^{-m} .
$$

Equating this to (2.34) gives the result.

Proof of Theorem 2. Using (2.21), the left hand side of (2.24) can be written as

$$
\begin{aligned}
L H S & =\sum_{1}^{\infty} \frac{1}{n \lambda^{n}}\left[\sum_{1}^{\infty} \frac{F_{m n}}{m \mu^{m}}\right]=\sum_{1}^{\infty} \frac{1}{n \lambda^{n}}\left[\mu^{n}-\mathcal{B}_{n}(\mu)\right] \\
& =\sum_{1}^{\infty} \frac{1}{n}\left(\frac{\mu}{\lambda}\right)^{n}-\sum_{1}^{\infty} \frac{1}{n \lambda^{n}} \mathcal{B}_{n}(\mu)=-\log \left(1-\frac{\mu}{\lambda}\right)-\sum_{1}^{\infty} \frac{Q_{n}(\mu)}{\lambda^{n}}
\end{aligned}
$$

On the other hand the right hand side of (2.24) is calculated as indicated in (2.25) and remarks thereafter. Thus

$$
\begin{aligned}
\text { RHS } & =\log \left[1-\frac{1}{\lambda-\mu} \sum_{1}^{\infty}\left(\frac{1}{\lambda^{n}}-\frac{1}{\mu^{n}}\right) \frac{F_{1 n}}{n}\right] \\
& =-\log (\lambda-\mu)+\log \left[\left(\lambda-\sum_{1}^{\infty} \frac{F_{1 n}}{n \lambda^{n}}\right)-\left(\mu-\sum_{1}^{\infty} \frac{F_{1 n}}{n \mu^{n}}\right)\right] \\
& =-\log (\lambda-\mu)+\log [P(\lambda)-P(\mu)] .
\end{aligned}
$$

This implies

$$
\log [P(\lambda)-P(\mu)]=\log \lambda-\sum_{1}^{\infty} Q_{n}(\mu) \lambda^{-n}
$$


which leads to (3.2). Then Lemma 2 implies the assertion.

Theorem 2 implies that the dispersionless Hirota equations (2.29) can be derived from the kernel formula (2.34) which is a direct consequence of the definition of $F_{m n}$ in (2.31). Thus Theorem 2 gives a direct proof of Theorem 1.

We will now express the $\chi_{n}\left(Q_{1}, \cdots, Q_{n}\right)$ as polynomials in $Q_{1}=P$ with the coefficients given by polynomials of $P_{j+1}$. First we have:

Lemma 3 One can write

$$
\chi_{n}=\operatorname{det}\left[\begin{array}{ccccccc}
P & -1 & 0 & 0 & 0 & \cdots & 0 \\
P_{2} & P & -1 & 0 & 0 & \cdots & 0 \\
P_{3} & P_{2} & P & -1 & 0 & \cdots & 0 \\
\vdots & \vdots & \vdots & \vdots & \vdots & \ddots & \vdots \\
P_{n} & P_{n-1} & \cdots & P_{4} & P_{3} & P_{2} & P
\end{array}\right]=\partial_{P} Q_{n+1} .
$$

Proof. In terms of $\chi_{n}\left(Q_{1}, \cdots, Q_{n}\right)=\partial_{P} Q_{n+1}$ the recursion relation (2.36) can be written in the form $\chi_{n}=P \chi_{n-1}+P_{2} \chi_{n-2}+\cdots+P_{n} \chi_{0}$. It is easy to derive the determinant expression from this form.

This leads to a rather evident fact, which we express as a proposition because of its importance. Thus:

Proposition 2 The $F_{m n}$ can be expressed as polynomials in $P_{j+1}=F_{1 j} / j$.

Proof. $\partial_{\lambda} Q_{n}=\chi_{n-1}\left(Q_{1} ; P_{2}, \cdots, P_{n-1}\right) \partial_{\lambda} Q_{1}\left(Q_{1}=P\right)$. Put this together with $Q_{n}=\mathcal{B}_{n} / n$ in (2.21) and $P$ in (2.17) to arrive at the conclusion (cf. also Remark 1 ).

Corollary 1 The dispersionless Hirota equations can be solved totally algebraically via $F_{m n}=\Phi_{m n}\left(P_{2}, P_{3}, \cdots, P_{m+n}\right)$ where $\Phi_{m n}$ is a polynomial in the $P_{j+1}$. Thus the $F_{1 n}=n P_{n+1}$ are generating elements for the $F_{m n}$.

This is of course evident from the dispersionless differential Fay identity (2.24). The point here however is to give explicit formulas of $F_{m n}$ in terms of the elementary Schur polynomials $\chi_{n}$ in (3.6).

Remark 1 One can also arrive at this polynomial dependence via the residue formulas of Lemma 1. As an adjunct to the proof of Proposition 2 we note now the following explicit calculations. Recall that $Q_{1}=P=\lambda-\sum_{1}^{\infty} P_{j+1} \lambda^{-j}$ and generally $Q_{n}=\lambda^{n} / n-\sum_{1}^{\infty} G_{j n} \lambda^{-j}\left(G_{j n}:=F_{j n} / j n\right)$. Thus from $Q_{2}=\lambda_{+}^{2} / 2=P^{2} / 2+P_{2}$ we get

$$
\frac{1}{2}\left(\lambda-\sum_{1}^{\infty} \frac{P_{j+1}}{\lambda^{j}}\right)^{2}+P_{2}=\frac{\lambda^{2}}{2}-\sum_{1}^{\infty} \frac{G_{2 j}}{\lambda^{j}} .
$$


Writing this out yields

$$
G_{2 m}=P_{2+m}-\frac{1}{2} \sum_{j+k=m} P_{j+1} P_{k+1} .
$$

This process can be continued with $Q_{3}=\lambda_{+}^{3} / 3=P^{3} / 3+P_{2} P+P_{3}$, etc.

It is also interesting to note the following relations:

Proposition 3 For any $n \geq 2$,

$$
\chi_{n}\left(-Q_{1}, \cdots,-Q_{n}\right)=-P_{n} .
$$

Proof. obtain (cf. (2.8))

$$
\left(\sum_{0}^{\infty} \frac{\chi_{n}(Q)}{\lambda^{n}}\right)\left(\sum_{0}^{\infty} \frac{\chi_{m}(-Q)}{\lambda^{m}}\right)=\sum_{0}^{\infty} \frac{1}{\lambda^{p}} \sum_{0}^{p} \chi_{p-k}(Q) \chi_{k}(-Q)=1 .
$$

Hence $\sum_{0}^{p} \chi_{p-k}(Q) \chi_{k}(-Q)=0$ for $p \geq 1$ which implies $\chi_{k}(-Q)=-P_{k}$ for $k \geq 2$ from the recursion relation (2.36).

Note from $P_{n+1}=F_{1 n} / n$ that the equations (3.9) give another representation of the dispersionless Hirota equations (2.29).

Remark 2 Formulas such as (3.6) and the statement in Proposition 2 indicate that in fact dKP theory can be characterized using only elementary Schur polynomials since these provide all the information necessary for the kernel (2.34) or equivalently for the dispersionless differential Fay identity. This amounts also to observing that in the passage from KP to dKP only certain Schur polynomials survive the limiting process $\epsilon \rightarrow 0$. Such terms involve second derivatives of $F$ and these may be characterized in terms of Young diagrams with only vertical or horizontal boxes. This is also related to the explicit form of the hodograph transformation where one needs only $\partial_{P} Q_{n}=\chi_{n-1}\left(Q_{1}, \cdots, Q_{n-1}\right)$ and the $P_{j+1}$ in the expansion of $P$ (cf. here (4.18)).

\section{Connections to D-bar}

It was shown in [6, 7, 14, 26] how inverse scattering information is connected to the dispersionless theory for KdV and some other situations (Benney equations and vector nonlinear Schrödinger equations for example). We will see here that although such connections seem generally not to be expected, nevertheless, one can isolate Dbar data for $S$ and $P$ in the dispersionless NKdV situations leading to an expression for the generating elements $F_{1 n}=n P_{n+1}$ which can be useful in computation. The technique also indicates another role for the Cauchy type kernel $1 /(P(\lambda)-P(\mu))$ in 
(2.34). Thus as background consider $\mathrm{KdV}$ and the scattering problem in the standard form (cf. [8])

$$
u_{t}=u^{\prime \prime \prime}-6 u u^{\prime} ; \quad \psi^{\prime \prime}-u \psi=-k^{2} \psi .
$$

Let $\psi_{ \pm}(k, x) \sim \exp ( \pm i k x)$ as $x \rightarrow \pm \infty$ be Jost solutions with scattering data, $T$ and $R$, determined via

$$
T(k) \psi_{-}(k, x)=R(k) \psi_{+}(k, x)+\psi_{+}(-k, x) .
$$

Setting $\psi_{-}=\exp (-i k x+\phi(k, x))$ we have from $(4.1)$

$$
v:=-\partial \log \left(\psi_{-}\right)=i k-\phi^{\prime} ; \quad \phi^{\prime \prime}-2 i k \phi^{\prime}+\phi^{2}=u \text {. }
$$

One looks for expansions,

$$
\phi^{\prime}=\sum_{1}^{\infty} \frac{\phi_{n}}{(i k)^{n}} ; \quad v=i k+\sum_{1}^{\infty} \frac{v_{n}}{(i k)^{n}},
$$

entailing $\phi_{n}=-v_{n}$. Here for example one can assume $\int_{-\infty}^{\infty}\left(1+x^{2}\right)|u| d x<\infty$ or $u \in \mathcal{S}$ $=$ Schwartz space for convenience so that all of the inverse scattering machinery applies. Then $T(k)$ will be meromorphic for $\operatorname{Im}(k)>0$ with (possibly) a finite number of simple poles at $k_{j}=i \beta_{j}\left(\beta_{j}>0\right),|R(k)|$ is small for large $|k|, k \in \mathbf{R}$, and $\log (T)=\sum_{0}^{\infty} c_{2 n+1} / k^{2 n+1}$ where $c_{n}$ can be written in terms of the $\beta_{j}$ and the normalization of the wave function $\psi_{ \pm}$. For $x \rightarrow \infty, \operatorname{Im}(k)>0, \psi_{-} \exp (i k x) \rightarrow 1 / T$ from (1.2), and taking logarithms $\phi(k, \infty)=-\log (T)$ which implies

$$
-\log (T)=\sum_{1}^{\infty} \frac{1}{(i k)^{n}} \int_{-\infty}^{\infty} \phi_{n} d x
$$

leading to $\int_{-\infty}^{\infty} \phi_{2 m} d x=0$ with $i^{2 m} c_{2 m+1}=\int_{-\infty}^{\infty} \phi_{2 m+1} d x$. Hence the scattering information $\left(\sim c_{2 n+1}\right.$, arising from $x$-asymptotics, given via $R$ and $\left.T\right)$ is related to the $k$-asymptotics $\phi_{n}$ of the wave function $\psi_{-}$. This kind of connection between space asymptotics (or spectral data - which is generated by these asymptotic conditions and forced by boundary conditions on $u$ here) and the spectral asymptotics of the wave function is generally more complicated and we refer to a formula in the Appendix to [6] describing the Davey-Stewartson (DS) situation

$$
-2 \pi i \chi_{n+1}=\int_{\Omega} \int \zeta^{n} \bar{\partial}_{\zeta} \chi d \zeta \wedge d \bar{\zeta}
$$

where $\bar{\partial}_{\zeta} \chi$ is D-bar data and the wave function $\psi$ has the form $\psi=\chi \exp (\Xi)$ with $\chi=1+\sum_{1}^{\infty} \chi_{j} \lambda^{-j}$ for $|\lambda|$ large (this is in a matrix form). The potentials in (4.6) occur in $\chi_{1}$. The D-bar data, or departure from analyticity of $\chi$, corresponds to spectral 
data in a sometimes complicated way and we refer to [1, 5, 6, 9, 11, 13, 16, 20, 22, 23. for more on this.

Now one expects some connections between inverse scattering for $\mathrm{KdV}$ and the $\mathrm{dKdV}$ theory since $\epsilon x=X$ means e.g. $x \rightarrow \pm \infty \sim \epsilon \rightarrow 0$ for $X$ fixed. Now from (4.2), we have

$$
\frac{1}{T}=\frac{1}{2 i k} W\left(\psi_{+}, \psi_{-}\right):=\frac{1}{2 i k}\left(\psi_{+}^{\prime} \psi_{-}-\psi_{+} \psi_{-}^{\prime}\right) .
$$

Given $\psi_{+} \sim \psi=\exp (S / \epsilon)$ and $\psi_{-} \sim \psi^{*}=\exp (-S / \epsilon)$ (cf. [6, 7]) one sees that $W\left(\psi_{+}, \psi_{-}\right) \rightarrow 2 \partial S / \partial X=2 P$, and from (2.17) with $\lambda=i k$,

$$
\frac{1}{T}=\frac{P}{i k}=1-\sum_{1}^{\infty} \frac{P_{n+1}}{(i k)^{n+1}} .
$$

Now $\log |T|$ and $\arg (R / T)$ have natural roles as action angle variables ( cf. [7]) and from $R=W\left(\psi_{-}(k, x), \psi_{+}(-k, x)\right) / W\left(\psi_{+}, \psi_{-}\right)$one obtains

$$
R=-\left(\frac{P(-k)+P(k)}{2 P(k)}\right) \exp \left(\frac{S(k)-S(-k)}{\epsilon}\right) \text {. }
$$

(We have suppressed the slow variables $T_{n}$ ). Thus the scattering data, which are considered to be averaged over the fast variables, now depend on the slow variables (in particular, see (4.5) and (4.8)). This corresponds to a dynamics of the Riemann surface determined by the scattering problem (the Whitham averaging approach). The exponential terms in (4.9) could in principle give problems here so we consider this in the spirit of [6, 17, 14]. Thus consider $\psi_{-}=\exp (-i k x+\phi)$ with $\phi(x)=$ $\int_{-\infty}^{x} \phi^{\prime}(\xi) d \xi$. Now $\psi_{-}$is going to have the form $\psi_{-}=\exp (-S(X, k) / \epsilon)$ and we can legitimately expect $-S / \epsilon=-i k X / \epsilon+\phi(X / \epsilon, k)$. For this to make sense let us write (note $\epsilon x=X, \epsilon \xi=\Xi)$

$$
\phi(X / \epsilon, k)=\int_{-\infty}^{X / \epsilon} \phi^{\prime}(\xi) d \xi=\frac{1}{\epsilon} \int_{-\infty}^{X} \phi^{\prime}(\Xi / \epsilon) d \Xi=\frac{1}{\epsilon}(i k X-S(X, k)) .
$$

Thus for $\phi(X / \epsilon, k)$ to equal $\epsilon^{-1}(i k X-S(X, k))$ we must have

$$
\int_{-\infty}^{X} \phi^{\prime}(\Xi / \epsilon) d \Xi=i k X-S(X, k)
$$

where $\phi^{\prime}=\partial \phi / \partial x$. This says that $\phi^{\prime}(\Xi / \epsilon)=f(\Xi / \epsilon)=\tilde{f}(\Xi)+O(\epsilon)$ which is reasonable in the same spirit that $u_{n}\left(T_{j} / \epsilon\right)=U_{n}\left(T_{j}\right)+O(\epsilon)$ was reasonable before. Thus we have

$$
P=\frac{\partial S}{\partial X}=i k-\phi^{\prime}=v ; \quad P^{2}-U=-k^{2},
$$

which corresponds to a dispersionless form of (4.3) in new variables. Note here that $\phi^{\prime \prime}=\epsilon \partial \phi^{\prime} / \partial X \rightarrow 0$ as $\epsilon \rightarrow 0$. Thus we have: 
Proposition 4 The manipulation of variables $x, X$ in (4.19) and (4.11) is consistent and mandatory. It shows that scattering information (expressed via $\phi$ for example) is related to the dispersionless quantity $S$ or $P$.

Further we have isolated D-bar data $\left(\bar{\partial}_{k} S\right)$ for $\mathrm{S}$ since for $P=\sqrt{U-k^{2}}$ one can write for arbitrary $X_{0}$ (cf. (4.11))

$$
i k X-S(X, k)=\int_{-\infty}^{X}(i k-P(\Xi, k)) d \Xi .
$$

Replace $-\infty$ by $X_{0}$ for large negative $X_{0}$ to get then $S=\int_{X_{0}}^{X} P d \Xi+i k X_{0}$. Thus one expects a pole for $|k| \rightarrow \infty$ plus D-bar data along $(-\sqrt{U}, \sqrt{U})$. This is in the spirit of [14, 15] where it is phrased differently. Observe that the expression $P=\sqrt{U-k^{2}}$ places us on a 2 sheeted Riemann surface with a cut along $(-\sqrt{U}, \sqrt{U})$ in the $k$ plane. Going around say $\sqrt{U}$ on the + sheet one has an opposite sides of the cut $P_{+}=i \sqrt{k^{2}-U}$ and $P_{-}=-i \sqrt{k^{2}-U}$, so $P_{+}-P_{-}=\Delta P=2 i \sqrt{k^{2}-U}$ which corresponds to D-bar data. This then leads to

$$
\Delta S=S_{+}-S_{-}=\int_{X_{0}}^{X} \Delta P d \Xi=2 \int_{X_{0}}^{X} \sqrt{U(\Xi)-k^{2}} d \Xi=2 \operatorname{Re} S_{+} .
$$

Note here that $P_{ \pm}$is real on the cut with $P_{-}=-P_{+}$(cf. Remark 3 for some general comments).

Consider next the situation of [6, 15, 18] with a reduction of dKP to

$$
\Lambda:=\lambda^{N}=P^{N}+a_{0} P^{N-2}+\ldots+a_{N-2},
$$

which is called dNKdV reduction. Assume $\Lambda(P)$ has $N$ distinct real zeros $P_{1}>$ $P_{2}>\ldots>P_{N}$ with $N-1$ interwoven turning points $\Lambda_{k}=\Lambda\left(\tilde{P}_{k}\right), P_{k+1}>\tilde{P}_{k}>P_{k}$. Assume, as $X \rightarrow-\infty$, these Riemann invariants $\Lambda_{k} \rightarrow 0$ monotonically so $\Lambda \rightarrow P^{N}$ with $(P-\lambda) \rightarrow 0$. Such situations are considered in [15] and techniques from [18 are adapted to produce formulas of the type $\left(W_{m}=\partial_{P} \mathcal{B}_{m}\right)$

$$
\begin{gathered}
S(X, \mu)-P(X, \mu) X-\mathcal{B}_{m}(P(X, \mu), X) T_{m} \\
=-\frac{1}{2 \pi i} \oint_{\Gamma} \frac{S(X, \lambda) \partial_{\lambda} P(X, \lambda)}{P(X, \lambda)-P(X, \mu)} d \lambda \\
\frac{\partial S}{\partial \mu}-\frac{\partial P}{\partial \mu}\left(X+W_{m}(P, X) T_{m}\right)=-\frac{1}{2 \pi i} \frac{\partial P}{\partial \mu} \oint_{\Gamma} \frac{S(X, \lambda) \partial_{\lambda} P(X, \lambda)}{(P(X, \lambda)-P(X, \mu))^{2}} d \lambda \\
=-\frac{\partial_{\mu} P}{2 \pi i} \oint_{\Gamma} \frac{\partial_{\lambda} S(X, \lambda)}{P(X, \lambda)-P(X, \mu)} d \lambda=\frac{\partial_{\mu} P}{\pi} \int_{\Gamma_{+}} \frac{\partial_{\lambda} \operatorname{Im} S(X, \lambda)}{P(X, \lambda)-P(X, \mu)} d \lambda .
\end{gathered}
$$


We see here the emergence of the Cauchy type kernel from (2.34) in an important role. At $\lambda_{k}:=\lambda\left(\tilde{P}_{k}\right)$ where $S_{\mu}$ is bounded and $P_{\mu} \rightarrow \infty$ (4.17) yields Tsarev type generalized hodograph formulas (cf. [15]). Thus such a formula is

$$
X+W_{m}\left(\tilde{P}_{k}(X), X\right) T_{m}=-\frac{1}{\pi} \int_{\Gamma_{+}} \frac{\partial_{\lambda} \operatorname{Im} S(X, \lambda)}{P(X, \lambda)-\tilde{P}_{k}(X)} d \lambda .
$$

Here one has Riemann invariants $\lambda_{k}=\lambda\left(\tilde{P}_{k}\right)$ where $\partial_{P} \Lambda=0$ and there is a collection $L$ of finite cuts through the origin of angles $k \pi / N(1 \leq k \leq N-1)$ in the $\lambda$ plane with branch points $\lambda_{k}$. One takes $\Gamma$ to be a contour encircling the cuts clockwise (not containing $\mu$ ) and sets $\Gamma=\Gamma_{-}-\Gamma_{+}$where + refers to the upper half plane. It turns out that $\left.S\right|_{\Gamma_{+}}=\left.\bar{S}\right|_{\Gamma_{-}}$and the contours can be collapsed onto the cuts to yield the last integral in (4.17) (see [11, 15] for pictures and details). By reorganizing the terms in the integrals one can express now integrals such as (4.17) in terms of D-bar data of $P$ on the cuts (cf. [11] for details). Thus $P$ and $S$ are analytic in $\lambda$ for finite $\lambda$ except on the cuts $L$ where there is a jump discontinuity $\Delta P$ (yielding $\Delta S$ by integration in $X$ as in (4.14)). We have seen for $\mathrm{dKdV}$ that $\Delta P=2 \sqrt{U-k^{2}}$ on $(-\sqrt{U}, \sqrt{U})$ and other $\mathrm{dNKdV}$ situations are similar (cf. [11, 15]). Moreover there is no need to restrict ourself to one time variable $T_{m}$ in (4.17), or for that matter to $\mathrm{dNKdV}$. Indeed the techniques, leading to formulas of the type (4.17) are based on 18] (cf. also [6]), and apply equally well to dKP provided the D-bar data $\bar{\partial} P$ for dKP lie in a bounded set $\Omega$ (cf. [1] and remarks below). In this respect, concerning dKP, one notes first that the transformation $t \rightarrow-t, U \rightarrow-U$ sends dKP-1 to dKP-2 so one expects that any D-bar data for $S$ or $P$ will be the same. Secondly, we know from [6, 18] that for large $|\lambda|$ there will be formulas of the type (2.34). Let us assume that $\bar{\partial} P$ (and hence $\bar{\partial} S$ ) is nontrivial only for a region $\Omega$ where say $|\lambda| \leq M<\infty$, and let $\Gamma$ be a contour enclosing $|\lambda| \leq M$. Then without regard for the nature of such data (Riemann- Hilbert data, poles, simple nonanalyticity, etc.) one can in fact derive formulas (4.17) following [6, 15, 18] (see [11] for details). We cannot collapse on cuts as in the last equation of (4.17) but we can think of the other formulas in (4.17) as integrals over D-bar data $\bar{\partial} P$ or $\bar{\partial} S$. It remains open to describe D-bar data for the dKP situations however. The idea of some kind of limit of spiked cut collections arises but we have not investigated this. In summary (cf. [11] for more detail) we can state:

Proposition 5 For $d N K d V$ (or dKP with given bounded D-bar data) one can write

$$
S(X, \mu)=P(\mu) X+\sum_{2}^{\infty} \mathcal{B}_{n}(P(\mu), X) T_{n}-\frac{1}{2 \pi i} \oint_{\Gamma} \frac{S(X, \lambda) \partial_{\lambda} P(X, \lambda)}{P(X, \lambda)-P(X, \mu)} d \lambda,
$$

where $\Gamma$ encircles the cuts (or D-bar data) clockwise. The determination of D-bar data here is to be made via analysis of the polynomial $\lambda^{N}$ in (4.15) for $d N K d V$ and in this situation one can again collapse (4.17) to the cuts. 
Remark 3 Now perhaps the main point of this has been to show that $S$ can be characterized via D-bar data of $S$ or $P$. In general we do not expect D-bar or scattering data for NKdV or KP to give D-bar data for $S$. The case of KdV is probably exceptional here and the situation of [14, 26] where spectral data for a system of nonlinear Schrödinger equations is related to the Benney or dKP hierarchy involves a different situation (the spectral data is not related to KP). Further since KP-1 and KP-2 have vastly different spectral or D-bar properties, and both pass to the same dKP, one does not expect the spectral data to play a role. We note also that spectral data is created by the potentials via asymptotic conditions for example (and vice versa) whereas D-bar data for dNKdV, arising from the polynomial $\lambda^{N}$, is a purely algebraic matter.

There is an interesting way in which D-bar data for $P$ or $S$ can be exploited. Thus, given that $S$ has D-bar data as indicated above in a bounded region, one can say that for $|\lambda|$ large and with some analytic function $A$ of $\lambda$,

$$
\begin{aligned}
S & =A+\frac{1}{2 \pi i} \iint \frac{\bar{\partial}_{\zeta} S}{\zeta-\lambda} d \zeta \wedge d \bar{\zeta} \\
& =A-\frac{1}{2 \pi i} \sum_{0}^{\infty} \frac{1}{\lambda^{j+1}} \iint \zeta^{j} \bar{\partial}_{\zeta} S d \zeta \wedge d \bar{\zeta}
\end{aligned}
$$

and from (2.19), we get

$$
\partial_{j} F=\frac{j}{2 \pi i} \iint \zeta^{j-1} \bar{\partial}_{\zeta} S d \zeta \wedge d \bar{\zeta}
$$

This is very useful information about $F_{j}$, since from the computation of (4.21) one could in principle compute all of the functions $F_{i j}$ for example. In fact, for $\mathrm{dKdV}$, and possibly some other $\mathrm{dNKdV}$ situations, one can obtain a direct formula for the $F_{1 j}$, which we know to generate all the $F_{i j}$ via Lemma 1 . Thus for $\mathrm{dKdV}$ we know from (4.14) that $\Delta S=\int_{X_{0}}^{X} \Delta P d X^{\prime}=2 \int_{X_{0}}^{X} \sqrt{U\left(X^{\prime}, T\right)-k^{2}} d X^{\prime}$ on the cut $L=(-\sqrt{U}, \sqrt{U})$ in the k-plane, and $\bar{\partial} S=\Delta S(i / 2) \delta_{L}$ where $\delta_{L}$ is a suitable delta function on $L$. Adjusting variables $\left(\zeta^{2}=-k^{2}\right.$ etc.) one can write then

$$
\partial F_{j}=-\frac{j(i)^{j-1}}{\pi} \partial \int_{-\sqrt{U}}^{\sqrt{U}} k^{j-1} \int_{X_{0}}^{X} \sqrt{U\left(X^{\prime}, T\right)-k^{2}} d X^{\prime} d k
$$

The only $X$ dependence here is visible and one can differentiate under the integral sign in (4.22). Since all $F_{12 m}=0$ by the residue formula (2.32), for example we take $j=2 n-1$ to obtain

$$
F_{12 n-1}=(-1)^{n} \frac{2 n-1}{\pi} \int_{-\sqrt{U}}^{\sqrt{U}} k^{2 n-2} \sqrt{U-k^{2}} d k=
$$




$$
=(-1)^{n} \frac{(2 n-1) U^{n}}{\pi} \int_{-\frac{\pi}{2}}^{\frac{\pi}{2}} \sin ^{2 n-2} \theta \cos ^{2} \theta d \theta=(-1)^{n}\left(\frac{U}{2}\right)^{n} \prod_{1}^{n} \frac{2 l-1}{l}
$$

(here $k=a \sin \theta$ and $\left.a^{2}=U\right)$. We summarize this in (cf. [11 for more detail)

Theorem 3 For any situation with bounded D-bar data for $S$ one can compute $F_{j}$ from (4.21). In the case of $d K d V$ with $\Delta S$ as indicated one can use (4.23) to determine directly the $F_{12 n-1}$ which will generate all the functions $F_{i j}$. Generally, if $\bar{\partial} S=$ $\int_{X_{0}}^{X} \bar{\partial} P\left(X^{\prime}, T, \zeta, \bar{\zeta}\right) d X^{\prime}$ (as in (4.29)), then from (4.21) $F_{1 j}=(j / 2 \pi i) \iint \zeta^{j-1} \bar{\partial} P d \zeta \wedge$ $d \bar{\zeta}$, and formally, $\partial_{n} \partial_{j} F=(j / 2 \pi i) \iint \zeta^{j-1} \bar{\partial} \mathcal{B}_{n} d \zeta \wedge d \bar{\zeta}$ since formally $\partial_{n} \bar{\partial} S=\bar{\partial} \partial_{n} S=$ $\bar{\partial} \mathcal{B}_{n}$.

Remark 4 The calculations using (4.22) in fact agree with the determination of $F_{12 n-1}$ from residue calculations as in Lemma 1 (note here $2 U_{2}=-U$ when adjusting notations between Sections 2 and 4). In general one may have some difficulties in determining $\bar{\partial} \mathcal{B}_{n}$ or $\bar{\partial} P$ for example, so the formulas (4.22) and (4.23) may be exceptional in this regard. However for $\mathrm{dKdV}$ we have checked the validity of these formal integrals for $F_{n j}$ involving $\bar{\partial} \mathcal{B}_{n}$ for a number of terms (e.g. $F_{31}, F_{51}, F_{33}, F_{35}$, and $\left.F_{53}\right)$ against the results of residue calculations. Here one can write for $n$ odd $\bar{\partial} \mathcal{B}_{n}=\bar{\partial} \zeta_{+}^{n}=\Delta \zeta_{+}^{n}=P^{-1} \zeta_{+}^{n} \Delta P$ and one gets for $n=2 k-1, j=2 m-1$

$$
F_{n j}=(-1)^{m} \frac{2(2 m-1) U^{k+m-1}}{\pi} \int_{0}^{\frac{\pi}{2}} \sin ^{2 m-2} \theta \hat{\zeta}_{+}^{2 k-1}(\cos \theta) \cos \theta d \theta
$$

where $a^{2 k-1} \hat{\zeta}_{+}^{2 k-1}(\cos \theta)=\zeta_{+}^{2 k-1}(P)$ for $P=a \cos \theta\left(a^{2}=U\right)$. Let us mention also that one can easily write down the tables of $F_{i j}$ for dNKdV from the table (2.30). Thus for $\mathrm{dNKdV}$ we have a reduction of the table (2.30) given via $F_{N m}=F_{m N}=0$ for all $m$.

Acknowledgment We thank Mr. Seung Hwan Son for his Mathematica programs yielding (2.30) from (2.26) and $F_{12 n-1}$ for the $\mathrm{dKdV}$ from (2.32). The work of $\mathrm{YK}$ is partially supported by an NSF grant DMS-9403597.

\section{References}

[1] M. Ablowitz and P. Clarkson, Solitons, nonlinear evolution equations and inverse scattering, Cambridge Univ. Press, 1991.

[2] M. Adler and P. vanMoerbeke, Comm. Math. Phys., 147 (1992), 25-56.

[3] S. Aoyama and Y. Kodama, Physics Lett. 295B, (1992), 190-198; Phys. Lett. 278B (1992), 56-62; Mod. Phys. Lett. 9A (1994), 2481-2492. 
[4] S. Aoyama and Y. Kodama, Topological Landau-Ginzburg theory with a rational potential and the dispersionless KP hierarchy, hep-th/9505122.

[5] M. Boiti, L. Martina, and F. Pempinelli, Multidimensional localized solitons, preprint, 1993.

[6] R. Carroll, Jour. Nonlinear Sci., 4 (1994), 519-544; Teor. i Matem. Fizika, 99 (1994), 220-225.

[7] R. Carroll, On dispersionless Hirota type equations, hep-th 9410063, Proc. NEEDS 94, World Scientific, to appear.

[8] R. Carroll, Topics in soliton theory, North-Holland, 1991.

[9] R. Carroll and B. Konopelchenko, Lett. Math. Phys., 28 (1993), 307-319.

[10] R. Carroll, Some connections between KdV and physics, in preparation.

[11] R. Carroll and S. Son, Remarks on dispersionless D-bar, in preparation.

[12] B. Dubrovin, Nucl. Phys. B, 374 (1992), 627-689; Comm. Math. Phys., 145 (1992), 195-207; Integrable systems, Birkhauser, 1993, pp. 313-359; hep-th 9303152; SISSA-89/94/FM, hep-th/9407018; Integrable quantum field theories, Plenum, 1993, pp. 283-302.

[13] A. Fokas and P. Santini, Physica 44D (1990), 99-130.

[14] V. Geogdzhaev, Teor. i Matem. Fizika, 73 (1987), 255-263; Singular limits of dispersive waves, Plenum, 1994, pp. 53-59.

[15] J. Gibbons and Y. Kodama, Singular limits of dispersive waves, Plenum, 1994, pp.61-66.

[16] J. Gibbons, Dynamical Problems in Soliton Systems, Springer, 1985, pp. 36-41.

[17] Y. Kodama, Phys. Lett. 129A (1988), 223-226 ; Prog. Theor. Phys., Supp. 94 (1988), 184-194.

[18] Y. Kodama and J. Gibbons, Phys. Lett. 135A (1989), 167-170; Proceedings IV Workshop on Nonlinear and Turbulent Processes in Physics, World Scientific, 1990, pp. 166-180.

[19] Y. Kodama, Lecture Lyon Workshop, July, 1991, unpublished. 
[20] B. Konopelchenko, Solitons in multidimensions, World Scientific, 1993; Introduction to multidimensional integrable systems, Plenum,1992.

[21] I. Krichever, Comm. Math. Phys., 143 (1992), 415-429; Comm. Pure Appl. Math., 47 (1994), 437-475; New symmetry principles in quantum field theory, Plenum, 1992, pp. 309-327.

[22] J. Leon, Jour. Math. Phys., 35 (1994), 3504-3524.

[23] S. Manakov, Physica 3D (1980), 149-157.

[24] K. Takasaki and T. Takebe, Inter. Jour. Mod. Phys. A, Supp., 1992, pp. 889-922.

[25] K. Takasaki and T. Takebe, hep-th 9405096.

[26] V. Zakharov, Funct. Anal. i Prilozh., 14 (1980), 15-24. 